\title{
Costos y calidad de la prueba de detección oportuna del cáncer cervicouterino en una clínica pública y en una organización no gubernamental
}

\author{
Jesica Gómez-Jauregui A., M. en Econ.S. ${ }^{(1)}$
}

\author{
Gómez-Jauregui A J. \\ Costos y calidad de la prueba de detección \\ oportuna del cáncer cervicouterino \\ en una clínica pública y en una organización \\ no gubernamental. \\ Salud Publica Mex 2001:43:279-288.
} El texto completo en inglés de este artículo está disponible en: http://www.insp.mx/salud/index.html

\section{Resumen}

Objetivo. Comparar los costos y la calidad de la prueba de detección oportuna del cáncer cervicouterino, entre una clínica pública y una organización no gubernamental (O N G). Material y métodos Se trata de un estudio de abordaje cuantitativo y cualitativo hecho en dos establecimientos asistenciales de la ciudad de Cuernavaca, Morelos, entre abril y julio de 1999. Mediante un estudio transversal se comparó el costo del proceso de producción de la prueba, desagregado en cuatro componentes, y para la evaluación de la calidad se utilizó el marco conceptual estructurado por Bruce, que incluye la percepción de las usuarias - acerca de la información recibida y el tiempo de espera- y de los proveedores -disponibilidad de espacio y de equipo-. Resultados LOS costos totales del proceso de producción completo de la clínica pública (144 pesos) fueron $26 \%$ más altos que los costos de la O N G (114 pesos). Por lo que se refiere a la calidad, las usuarias de la O N G informaron una mayor satisfacción con los servicios recibidos. La inconformidad de las mujeres que se realizaron la prueba en la clínica pública se relacionó con el tiempo de espera para la toma de la muestra y para la entrega de los resultados. Conclusiones Las diferencias en los costos y los indicadores de calidad de la atención entre ambos proveedores sugieren que las 0 NG deben contemplarse como una al-

\section{Gómez-Jauregui A J.}

Comparison of Cervical Cancer

Screening Program costs and quality

of care between a public clinic and a Non-Governmental

Organization.

Salud Publica Mex 2001;43:279-288.

The English version of this paper

is available at: http://www.insp.mx/salud/index.html

\begin{abstract}
A bstract
Objective. To compare the costs and quality of cervical cancer screening between a non-governmental organization (N GO) and a Ministry of $\mathrm{H}$ ealth clinic. Material and methods. A quantitative and qualitative cross-sectional study was conducted between A pril and July 1999, in two healthcare clinics in C uernavaca, Morelos, Mexico. Cervical cancer screening production processes were compared along four components: a) Pap smear collection; b) its transportation to the cytology (enter; $c$ ) analysis and interpretation; and d) notification of results. The framework developed by Bruce was used for assessing the quality of care.The framework includes users' perceptions on information received and waiting times, as well as providers' perceptions of space and equipment availability. Results. The unitary cost of the production process in the public clinic ( $\$ 144$ pesos or US\$15.5) was $26 \%$ higher than in the NGO (\$114 pesos or US\$12.3). W omen attending N GO services reported a higher satisfaction with the quality of care than those who attended the public clinic.The waiting time prior to screening and the time each woman has to wait to receive test results were the main sources of insatisfaction. Conclusions. Analysis of costs and quality of care results suggest that $\mathrm{NGO}$ s should be considered as an alternative in the provision of cervical cancer screening. The English version
\end{abstract}

(1) Centro de Investigación en sistemas de Salud, Instituto N acional de Salud Pública, México.

Fecha de recibido: $\mathbf{2 8}$ de septiembre de $\mathbf{2 0 0 0}$ • Fecha de aprobado: 7 de marzo de 2001

Solicitud de sobretiros: M. Jesica Gómez-Jauregui A. Centro de Investigación en Sistemas de Salud, Instituto N acional de Salud Pública. A venida Universidad 655, colonia Santa María A huacatitlán, 62508 Cuernavaca, Morelos, México.

Correo electrónico: jesica@ datasys.com.mx 
ternativa para ofrecer servicios de detección oportuna de cáncer cervicouterino. El texto completo en inglés de este artículo está disponible en: http://www.insp.mx/salud/ index.html

Palabras clave: neoplasmas del cuello uterino; citodiagnóstico; análisis de costos; calidad de la atención; organización no gubernamental; M éxico of this paper is available at: http://www.insp.mx/salud/ index.html

Key words: cervix neoplasms; cytodiagnosis; costs analysis; quality of care; non-governmental organizations; M exico
E 1 cáncer cervicouterino $(\mathrm{CaCu})$ es la forma más común de cáncer entre las mujeres de los países en desarrollo y es, después del cáncer de mama, el segundo tipo más común de cáncer en el mundo. ${ }^{1}$ Por esta razón se han creado programas específicos de detección oportuna. Estos programas han sido particularmente exitosos en Dinamarca, Islandia, Finlandia, Suecia, el Reino Unido, Canadá y Estados Unidos de América (EUA). ${ }^{2}$ En otros países no han tenido el éxito esperado, debido, en gran parte de los casos, a la implantación inadecuada de las políticas.

La prueba que se utiliza comúnmente para detectar este tipo de cáncer es la técnica de Papanicolaou, desarrollada en los años treinta. El objetivo de esta prueba es detectar, en un estadío temprano, células neoplásicas. Es en estas etapas cuando se puede utilizar con éxito un procedimiento quirúrgico de relativo bajo costo y bajo riesgo, que permite remover dichas células y prevenir la diseminación del cáncer. ${ }^{3}$

Los estudios realizados en países desarrollados coinciden en señalar que los programas de detección que utilizan la prueba de Papanicolaou son eficientes y costo-efectivos. De hecho, Brown y Garber ${ }^{4}$ encontraron que el aumento reciente en la sensibilidad de la prueba de Papanicolaou la ha hecho todavía más costo-efectiva. Koopmanschap y colaboradores, ${ }^{5}$ en un estudio realizado en Holanda, concluyeron que la detección oportuna es una acción costo-efectiva si se la compara con no realizarla y tener que atender a una mujer con $\mathrm{CaCu}$ avanzado. Mandelblatt y Fahs, ${ }^{6}$ por su parte, realizaron un estudio en mujeres mayores de 65 años, de bajos ingresos, en EUA, y concluyeron, también, que el programa de detección oportuna en esta población disminuye los costos de la atención y ahorra recursos en el futuro.

En México, la prueba de Papanicolaou comenzó a utilizarse en 1949 en el Hospital de la Mujer. Sin embargo, su uso masivo se inició en 1974 con el Programa Nacional de Detección Oportuna de Cáncer (PNDC). No obstante, el CaCu sigue siendo uno de los principales problemas de salud pública del país. Entre 1985 y 1995, la tasa de mortalidad por CaCu en Mé- xico se incrementó de 7.7 por 100000 mujeres a $9.6,{ }^{7}$ la cual es una de las tasas de mortalidad por CaCu más altas del mundo. ${ }^{8}$ En el estado de Morelos, la tasa de mortalidad por esta causa es de 16.0/100 000 mujeres. $^{9}$ No es de extrañar que $48 \%$ de las mujeres de este estado nunca se hayan realizado la prueba de Papanicolaou. ${ }^{10}$

En 1996, los recursos dedicados a prevenir este tipo de padecimientos alcanzaron para realizar 3316000 pruebas de Papanicolaou, destinadas a una población de 16 millones y medio de mujeres de entre 25 y 65 años de edad. ${ }^{11}$ Esta cantidad resulta insuficiente si se considera que la Norma Oficial Mexicana recomienda una prueba de detección, cada tres años, para las mujeres que presentan resultados negativos en sus dos citologías anuales previas. ${ }^{12}$ Actualmente, la prevalencia del uso del Papanicolaou en México es de 53\%, existen entonces ocho y medio millones de mujeres que nunca se han realizado la prueba, y dos y medio millones que se realizaron la prueba al menos tres años atrás. Esta cifra supone que $46.7 \%$ de las mujeres que afirman haberse realizado la prueba lo hace al menos cada tres años.

Además de la falta de recursos, el PNDC enfrenta el problema de la baja utilización de sus servicios, debido entre otros hechos, a barreras culturales y geográficas, al mal trato que reciben las mujeres por parte del personal de salud, a la mala calidad de las muestras y de su interpretación, y a la baja notificación de los resultados. ${ }^{12-14}$

Así como la Secretaría de Salud (SSA) considera a la mortalidad por $\mathrm{CaCu}$ como uno de los problemas prioritarios de la salud pública de México, existen otras organizaciones que no pertenecen al gobierno (ONGs) que, conscientes de este problema, han puesto en marcha programas propios de detección oportuna de este tipo de cáncer dentro del marco de programas de salud dirigidos a la mujer. Uno de los grupos pioneros en este sentido es CIDHAL (Comunicación, Intercambio y Desarrollo Humano en América Latina), una ONG ubicada en Cuernavaca, Morelos, que cuenta con una programa de detección oportuna de $\mathrm{CaCu}$. 
El programa se realiza con dos modalidades: dentro y fuera del consultorio. En el primer caso, la toma de la citología se hace en el consultorio y el resultado se entrega allí mismo. En el segundo caso, la toma se efectúa en la casa de alguna de las parteras que colaboran con CIDHAL, o en alguna clínica de salud de la SSA, con la que existe algún acuerdo de cooperación.

Puesto que las instituciones públicas han enfrentado serios problemas en su intento por disminuir la mortalidad por $\mathrm{CaCu}$, y dado que existen ONGs que también están participando en esta lucha, puede ser útil evaluar la posibilidad de que este tipo de organizaciones ofrezcan servicios de esta naturaleza más amplios. El objetivo de este estudio, de hecho, es comparar los costos y la calidad de la prueba de detección oportuna de $\mathrm{CaCu}$ entre una clínica de primer nivel de atención, de la SSA de Cuernavaca, Morelos, y CIDHAL, para determinar qué proveedor cuenta con un programa más eficiente y de mayor calidad.

\section{Material y métodos}

Se trata de un estudio de caso de corte transversal, en el que se comparan los costos y la calidad de la prueba de detección oportuna de $\mathrm{CaCu}$ entre dos organismos prestadores de ese servicio: una clínica pública de primer nivel de atención, ubicada en la ciudad de Cuernavaca, Morelos, México y CIDHAL, una ONG ubicada en esa misma ciudad, que también proporciona servicios de salud de primer nivel de atención principalmente a población femenina de bajos recursos. El universo muestral para ambas unidades lo conforman todas las mujeres evaluadas durante visitas a las clínicas, y que recibieron ya los resultados de su estudio. Para corregir la diferencia que pudiera existir en la incidencia de cáncer cervicouterino en la población que atiende cada una de las unidades, se utiliza el siguiente:

Porcentaje de mujeres con prueba

Factor de corrección $\frac{\text { positiva que fueron notificadas }}{\text { Total de mujeres que }}$ se realizaron la prueba

El proceso de producción de la prueba de detección oportuna del cáncer incluyó cuatro componentes: a) la toma de la muestra citológica, b) el traslado de la muestra al laboratorio donde se analizó, c) el proceso de tinción e interpretación y d) la notificación de resultados. La función de producción se determinó después de consultar a un grupo de expertos sobre los pasos que se siguen en la prueba de detección oportuna de este tipo de cáncer. Para determinar el proceso particular de la clínica de la SSA y de CIDHAL, se entrevistó al personal médico a cargo de ambas unidades. Se entrevistó también a personal de salud de varias categorías y a las usuarias del servicio, para verificar la existencia de los equipos, materiales y procedimientos reportados en la primera entrevista.

Los costos totales se integraron mediante la suma de los costos de los cuatro componentes mencionados. Para los costos directos se calculó el costo unitario de operación, y para los costos indirectos, el costo promedio. Es importante distinguir entre dos conceptos de costos: el costo financiero o contable y el costo económico. Los costos financieros son el dinero asignado a los recursos necesarios para producir la intervención, los costos económicos son los costos de oportunidad de los recursos utilizados al llevar a cabo la intervención, es decir, el beneficio que se obtendría en el mejor uso alternativo. Es importante, en términos de una evaluación económica, incluir en su totalidad el valor de los recursos, por eso es conveniente utilizar el costo económico de cada una de las intervenciones a comparar. Asimismo, se calcula el costo unitario para los costos directos, ya que el procedimiento, tipo y cantidad de insumos no varía para cada mujer. En el caso de los costos indirectos, el tiempo de transporte y de espera sí varían, por lo que se decidió calcular el costo promedio de esos recursos. ${ }^{15}$ Los costos incluidos en cada uno de los cuatro componentes se describen en el cuadro I. La estructura de las matrices utilizadas para estimar el costo se basan en la propuesta de Arredondo y colaboradores. ${ }^{15}$

Los costos de infraestructura, administración y servicios a la unidad (agua, luz) no se incluyen en el estudio, ya que no existe diferencia en su utilización entre las dos unidades; es decir, el espacio, la administración y la cantidad de agua y de luz que se utilizan son reducidos y muy similares en ambos establecimientos.

Los costos de la clínica de primer nivel de atención se obtuvieron de las adquisiciones realizadas mediante el mecanismo de compra consolidada de la SSA. Los costos de los insumos empleados en CIDHAL se obtuvieron aplicando el precio de mercado. En cuanto a los recursos humanos, en el caso de la SSA se consideraron los salarios asignados por esta misma; para CIDHAL, que cuenta con personal voluntario, se utilizó el precio de mercado de esa actividad. ${ }^{16}$ Con el fin de determinar el costo del mobiliario, equipo e instrumental, se calculó el costo anual equivalente. ${ }^{17}$

Los costos indirectos incluyeron el tiempo de espera en la unidad antes de realizar la prueba y el tiempo de transporte del hogar a la clínica. El costo del tiempo es el costo de oportunidad de la espera en la unidad y del transporte -equivalentes al salario que se deja de ganar por esperar y transportarse. Como una 


\section{Cuadro I

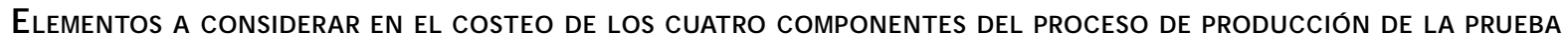 de detección oportuna de cáncer Cervicouterino. Cuernavaca, Morelos, México, 1999}

\begin{tabular}{lccc}
\multicolumn{2}{c}{ Costos por componente } & \\
Toma de muestra & Traslado al laboratorio & Laboratorio & Resultados \\
& & & Costos directos \\
Costos directos & Costos directos & Recursos humanos \\
Instrumental e insumos & Costo de traslado & Costos directos & Mobuipo \\
Equipo & Instrumentos de traslado & Instrumental & Substancias químicas \\
Recursos humanos & & Recursos humanos \\
Mobiliario & Pago de bolsillo (CID HAL) \\
Pago de bolsillo de usuarias (CID HAL) & & \\
Costo de traslado de instrumental (segunda & & Costos indirectos \\
modalidad de CID HAL) & Tiempo de traslado \\
Costos indirectos & & Tiempo de espera \\
Tiempo de traslado a la clínica (paciente) & & \\
Tiempo de espera & & \\
\end{tabular}

aproximación, se estimó el salario promedio de las mujeres entrevistadas de acuerdo con la ocupación reconocida.

Además, se realizó un análisis de sensibilidad con los costos indirectos y con la tasa de descuento. En los dos casos se trata de parámetros imprecisos. En el primer caso, el tiempo de espera y de traslado reportado por las mujeres es variable. En el segundo, la tasa de descuento utilizada para estimar costos de capital varió del 3 al 10\%.

Para CIDHAL, se realizó el costeo de la toma de la muestra dentro y fuera del consultorio, según la modalidad de atención. Conviene señalar que las tomas en zonas marginadas impactan de manera positiva el nivel de calidad de la atención, incrementan la cobertura, aumentan el porcentaje de nuevas usuarias al programa y facilitan el acceso. La única variación en los insumos de las dos modalidades se presentó en el costo del traslado de personal y de materiales al lugar donde se efectúa la toma.

La metodología para evaluar la calidad del programa de detección oportuna del $\mathrm{CaCu}$ se basó en el marco conceptual, estructurado por Bruce, para estimar la calidad de los servicios de planificación familiar. ${ }^{18}$ La autora asegura que mejorar la calidad tiene impacto en el uso del programa, es decir, a mejor calidad, mayor constancia en las visitas de las usuarias para realizarse la prueba de detección oportuna del $\mathrm{CaCu}$ y mayores las recomendaciones a otras mujeres. De acuerdo con Stein, ${ }^{19}$ la propuesta metodológica de Bruce debe reforzarse proporcionando información que vincule la percepción de las mujeres sobre la calidad del servicio con la calidad técnica. Esto se puede lograr mediante el uso de entrevistas de salida y estudios sombra. En este caso se decidió añadir entrevistas a los proveedores para determinar la relación usuarioproveedor desde la perspectiva tanto del proveedor como del consumidor. Además, se realizó un estudio sombra para validar la información de las entrevistas, tanto de las usuarias como del personal de salud.

Para evaluar el nivel de calidad de la intervención se utilizaron tres componentes: a) calidad desde la perspectiva de las usuarias (percepción de la calidad del servicio por parte de las usuarias; información proporcionada a las usuarias relacionada con la prueba de detección; tiempo de espera de los resultados y opinión de las usuarias al respecto, y disponibilidad de equipo e insumos); b) calidad desde la perspectiva de los proveedores (ventajas y limitaciones del programa; información proporcionada a las usuarias y control de calidad de la toma e interpretación de las citologías), y c) calidad desde la perspectiva del entrevistador (trato a las usuarias; promoción e información proporcionada a las usuarias y disponibilidad de equipo e insumos).

Para determinar la percepción de las usuarias sobre la calidad de la atención se aplicó un cuestionario a 30 mujeres (15 de cada unidad), seleccionadas aleatoriamente de entre las usuarias de la SSA y CIDHAL. Para conocer la opinión del personal de salud sobre la calidad y las condiciones de la atención, se realizaron entrevistas al personal operativo. En la clínica de la SSA se entrevistó a dos enfermeras generales y a la médica a cargo de la clínica. En CIDHAL se entrevistó a dos parteras que colaboran con la organización y a la médica que dirige el área de salud. El entrevistador 
verificó la información y observó, detalladamente, el proceso que siguen ambos proveedores en el programa de detección de $\mathrm{CaCu}$.

\section{Resultados}

La información general obtenida de las mujeres entrevistadas es la siguiente: nivel de escolaridad, ocupación y edad. En cuanto a escolaridad, $30 \%$ de las mujeres que acudieron al programa de CIDHAL en su modalidad fuera del consultorio, y $40 \%$ de las mujeres que acudieron a la SSA terminaron la primaria. Debe señalarse que todas las mujeres estudiaron al menos un año de primaria. La escolaridad de las mujeres que asisten a realizarse la prueba en el consultorio de CIDHAL, varía desde tener la primaria sin terminar hasta estudios de posgrado. Se trata de población mucho más heterogénea. La ocupación de las mujeres de la SSA y CIDHAL fuera del consultorio es muy similar, en ambos casos $80 \%$ se dedican al hogar. La distribución de la edad de las mujeres entrevistadas tiene una media de 33 años (con 10\% mayor a 45 años) en la SSA y de 44 años (60\% mayor de 45 años) en CIDHAL.

Los resultados obtenidos no son estadísticamente significativos, ya que el tamaño de la muestra es muy pequeño. Se trata de un estudio piloto para probar la factibilidad de utilizar un modelo de evaluación económica, y comparar así una misma intervención realizada por proveedores de dos sectores distintos.

\section{Costos}

Para ambos proveedores se estimaron los costos promedio de cada una de las cuatro etapas del proceso de producción. Así, el costo unitario por usuaria de la toma de citología, desde la perspectiva del productor, fue de 38 pesos para la SSA y de 38.7 pesos para CIDHAL (producir la toma cuesta 47 pesos, menos el pago de bolsillo que realiza la usuaria, que es de 8.5 pesos). El costo unitario para las usuarias, considerando el tiempo promedio de espera y de traslado (el tiempo promedio de espera para la SSA es de 65 minutos y el de traslado de diez minutos; para CIDHAL el tiempo promedio de espera es de 21 minutos, y el de traslado, de ocho minutos), fue de 16 pesos para las usuarias de los servicios de la SSA y de 14.5 pesos para las usuarias de CIDHAL.

Por lo que se refiere a la segunda modalidad de la toma de citología de CIDHAL -fuera del consultorio-, el costo de producción de la toma, añadiendo el costo del traslado del personal de CIDHAL y del material, fue de 40.8 pesos. El pago de bolsillo de las usuarias de CIDHAL varía de 8.5 a 300 pesos. No obstante, la población de bajo nivel socioeconómico que utiliza los servicios de CIDHAL, y que es comparable con la de la clínica de primer nivel de atención de la SSA, paga la tarifa más baja, 8.5 pesos, y en algunos casos no realiza pago alguno (el pago en este caso es en especie, con arroz, frijol o aceite).

Los costos indirectos, que incluyen el costo promedio del tiempo de traslado y de espera de las usuarias, se calcularon sobre la base del salario de una empleada doméstica, ya que el $80 \%$ de las usuarias reconocieron ser amas de casa.

El costo unitario del traslado de las laminillas al laboratorio fue de 27.08 pesos para la SSA y de 26.78 pesos para CIDHAL.

El costo unitario de tinción e interpretación de las citologías fue de 35.10 pesos para el laboratorio de la SSA y de 22.12 pesos para el laboratorio privado (CIDHAL debe realizar un pago de 30 pesos por laminilla al laboratorio. Al restar al pago de bolsillo que realiza CIDHAL el costo de tinción e interpretación, el laboratorio obtiene una ganancia de ocho pesos).

El costo unitario de la entrega de resultados fue de 12.6 pesos para la SSA y de 6.2 pesos para CIDHAL. Por su parte, el costo unitario fue de 15.6 pesos para las usuarias de los servicios de la SSA y de seis pesos para las de CIDHAL (ambos, estimados con un tiempo de espera y de transporte promedio).

Los costos totales de los proveedores fueron de 112.6 pesos para la SSA y de 101.7 pesos para CIDHAL. Los costos totales para cada usuaria fueron de 31 pesos para las mujeres que acuden a la SSA y de 20.5 pesos para las mujeres que se atienden en CIDHAL (figura 1).

Así, los costos totales, desde la perspectiva social (sin considerar las transferencias o pago de bolsillo de las usuarias a CIDHAL y de CIDHAL al laboratorio), fueron de 143.8 pesos para la SSA y de 114 pesos para CIDHAL (cuadro II).

En el análisis de sensibilidad se varió la tasa de descuento utilizada para estimar el costo anual equivalente del mobiliario, equipo e instrumental, de $3 \%$ a $10 \%$, con lo que los costos de estos rubros se incrementaron en $34 \%$ en ambas unidades. Los costos indirectos, tiempo de espera y tiempo de transporte, variaron de acuerdo con la información proporcionada por las usuarias. Así, los costos indirectos en la SSA se ubicaron en 4.79 pesos, con 20 minutos de espera y tres minutos de traslado, y 27.08 pesos, con 120 minutos de espera y diez minutos de traslado. En CIDHAL, los costos indirectos variaron de 3.54 pesos, con dos minutos de traslado y 15 minutos de espera, a 11.45 pesos, con 15 minutos de traslado y 40 minutos de espera. 


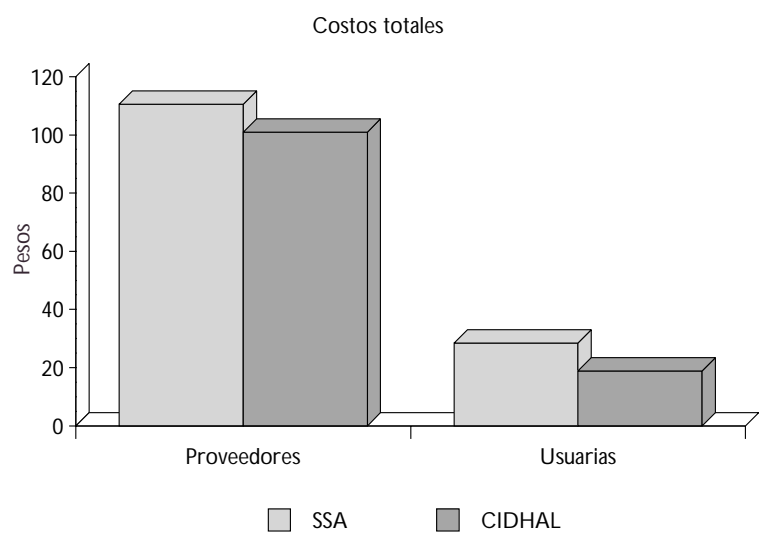

Suma de los costos de toma de la muestra, traslado de laminillas al laboratorio, tinción e interpretación de la muestra y entrega de resultados.

Figura 1. Costos totales promedio del proceso de PRODUCCIÓN DE LA PRUEBA DE DETECCIÓN OPORTUNA DEL Cáncer cervicouterino. Cuernavaca, Morelos, MéXICO, 1997

Cuadro II

Costo total PROMEDIO DESDE LA PERSPECTIVA SOCIAL DE LA PRUEBA DE DETECCIÓN OPORTUNA DEL CÁNCER CERVICOUTERINO. Cuernavaca, Morelos, México, 1999

\begin{tabular}{lcccc} 
& $\begin{array}{c}\text { SSA } \\
\text { (pesos) }\end{array}$ & $\begin{array}{c}\text { CIDHAL } \\
\text { (pesos) }\end{array}$ & $\%$ \\
Recursos humanos & 84.3 & 58.6 & 69.4 & 60.8 \\
\hline Aparatos y mobiliario & 10.5 & 7.3 & 1.58 & 1.4 \\
\hline Instrumental y recipientes & 1.54 & 1.07 & 1.13 & 1 \\
\hline Material desechable y otros insumos & 7.1 & 4.9 & 26.1 & 23 \\
\hline Papelería & 3.5 & 2.4 & 2.1 & 1.8 \\
\hline Transporte & 5.7 & 3.9 & 1.9 & 1.6 \\
\hline Tiempo de transporte y espera & 31.2 & 21.6 & 12 & 10.5 \\
\hline Total & 143.8 & 100 & 114 & 100
\end{tabular}

Los tiempos promedio de espera y de traslado para la SSA fueron de $65 \mathrm{y}$ diez minutos, respectivamente, con un costo de 15.6 pesos. Para CIDHAL los tiempos promedio de espera y traslado ascendieron a 21 y ocho minutos, respectivamente, con un costo de seis pesos.

\section{Calidad}

Percepción de las usuarias

En relación con la información proporcionada por ambos proveedores sobre los riesgos de desarrollar cáncer cervicouterino, y sobre la importancia de realizarse la prueba de detección, $70 \%$ de las usuarias de la SSA y el $100 \%$ de las de CIDHAL consideran como suficiente esa información.

En cuanto a la calidad de la atención recibida, $60 \%$ de las mujeres que acudieron a la clínica de la SSA y el $100 \%$ de las que acudieron a CIDHAL, informaron estar muy satisfechas con la atención proporcionada por el personal de salud. En ningún caso hubo mujeres que expresaran estar totalmente insatisfechas.

La falta de espacio o equipo necesario para realizar la prueba es una limitación al programa: 80 \% de las usuarias de la SSA reportaron un espacio limitado en la clínica, tanto para esperar como para tomar las muestras. En cuanto al equipo, 50\% reportaron escasez de material, ya que en muchas ocasiones ellas deben comprar los guantes de látex. Sólo el 10\% de las usuarias de CIDHAL reportaron que el espacio, cuando se realiza la toma fuera de la clínica, es limitado. En cuanto al equipo, ninguna reportó que éste fuera insuficiente.

Una de las características que más impacta a la percepción de las usuarias de la calidad de algún servicio es el tiempo que tienen que esperar antes de recibirlo. En el caso de la SSA el tiempo de espera reportado varió de 15 minutos a dos horas.; $60 \%$ de las mujeres mencionaron haber esperado más de media hora. En CIDHAL el tiempo de espera informado varió de 15 a 40 minutos; $13 \%$ esperó más de media hora.

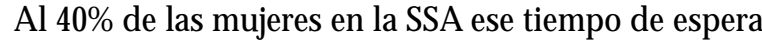
les pareció demasiado. Sólo al 10\% de las usuarias de CIDHAL les pareció mucho el tiempo de espera. En cuanto al tiempo para recibir los resultados de la prueba, éste varió en la SSA de dos a tres meses y en CIDHAL de ocho a 15 días. De las mujeres que acuden a la SSA, $70 \%$ consideró que ese es mucho tiempo; en contra parte, ninguna de las mujeres de CIDHAL lo consideró como demasiado (figura 2).

$\mathrm{Al}$ preguntarles si recomendarían ese servicio a alguna mujer miembro de la familia o a alguna amiga, el $60 \%$ de las entrevistadas en la clínica de la SSA respondieron que sí lo recomendarían; el otro $40 \%$ expuso como razón para no recomendar el servicio el tiempo tan largo que deben esperar para recoger sus resultados. Las mujeres que utilizaron el servicio de CIDHAL respondieron en su totalidad que sí lo recomendarían.

\section{Percepción de los proveedores}

De acuerdo con la información proporcionada, en la SSA son las enfermeras las que toman la citología y sólo se llama a la médica en caso de encontrar alguna anormalidad; no obstante, es la médica quien entrega 
los resultados, sean positivos o negativos. En CIDHAL, tanto las parteras como la médica realizan las tomas de citologías y entregan resultados. Sobre la capacitación para promoción y para una correcta obtención de la citología, el personal de la SSA expresó que es necesario recibir una mayor capacitación y una constante actualización sobre el $\mathrm{CaCu}$, su tratamiento y prevención. Por su parte, las parteras que colaboran con CIDHAL respondieron que constantemente reciben capacitación, por parte de la organización, en la toma de muestras y en la promoción y orientación del programa.

Al preguntar sobre la disponibilidad de material y espacio, el personal de la SSA coincidió en que el sitio en la clínica es insuficiente, ya que no se cuenta con un espacio exclusivo y privado para la toma de citologías, sólo existe una habitación destinada, tanto a la realización de la prueba como a vacunación, control prenatal y curaciones. En relación a la disponibilidad de insumos, es común que no reciban dentro del material que les surten la cantidad necesaria de guantes de látex, por lo que tienen que pedirlos a las usuarias.

Las parteras que colaboran con CIDHAL no reportaron limitación en el espacio debido a que éste se ajusta a las necesidades del trabajo que se realiza fuera del consultorio. Del mismo modo, el espacio con el que cuenta el consultorio es suficiente. En cuanto a los insumos, la médica responsable del área de salud mencionó que en ocasiones la falta de recursos limita el número de tomas, debido a que CIDHAL no es una organización lucrativa y obtiene gran parte de sus re-

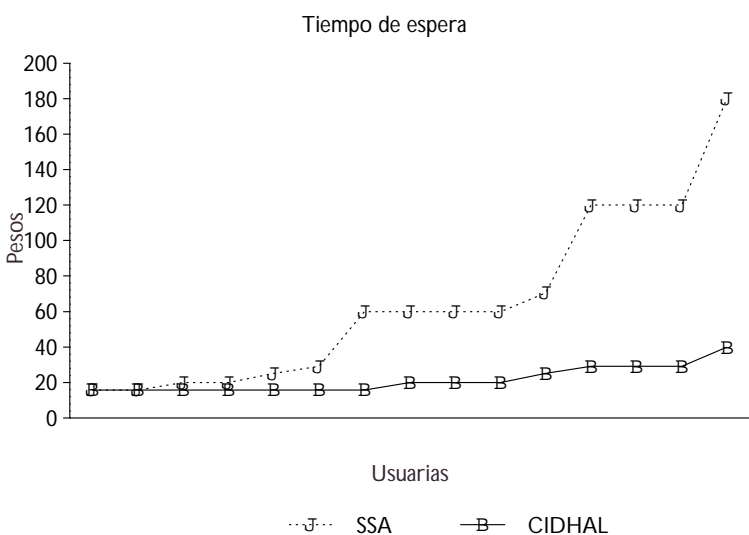

Figura 2. TIEMPO de espera de LAS USUARIAS PARA LA TOMA DE MUESTRA, ENTREGA DE RESULTADOS DE LA PRUEBA DE DETECCIÓN OPORTUNA DEL CÁNCER CERVICOUTErino. Cuernavaca, Morelos, MéXico, 1999 cursos de organismos internacionales, los cuales no otorgan dinero de manera permanente ni a una sola organización. No obstante, CIDHAL efectuó un número mayor de pruebas para 1998 (figura 3).

El proceso reportado del control de calidad de la toma, tinción e interpretación de la citología, es el siguiente:

En la SSA no existe contacto entre el personal de la clínica y el personal del laboratorio. Las citologías se llevan cada semana a la jurisdicción sanitaria correspondiente y el personal de ésta se encarga de llevarlas al laboratorio. El control sobre la calidad de la toma lo realiza el laboratorio. En caso de que la laminilla no cuente con las características necesarias para su tinción e interpretación, se pide a la clínica que practique una segunda toma de la citología. Para determinar el control de calidad del mecanismo de tinción e interpretación, se entrevistó al personal del laboratorio central del Hospital General de México de la SSA. No se tuvo acceso al laboratorio de la SSA en Cuernavaca donde se procesan las muestras de citología de la clínica de primer nivel de atención; suponemos, entonces, que el proceso que se sigue en ambos laboratorios es muy similar, por ajustarse a los lineamientos que establece la Secretaría de Salud en la Prueba de Papanicolaou. De acuerdo a su informe, el laboratorio cuenta con controles interno y externo. El primero consiste en elegir una muestra aleatoria del $2 \%$ de las laminillas ya interpretadas para una nueva interpretación por parte de los patólogos. El segundo consiste en una evaluación periódica sobre las habilidades de citotecnólogos y patólogos en la interpretación de las

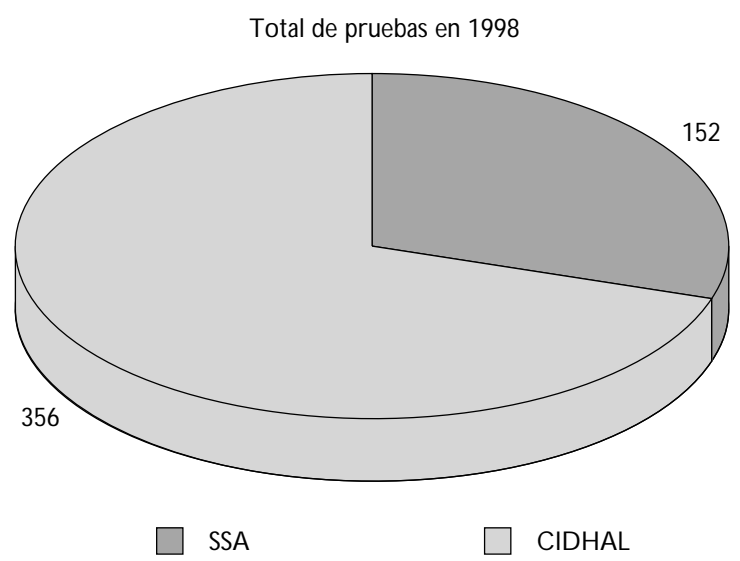

Figura 3.Total de pruebas de detección oportuna DE CÁNCER CERVICOUTERINO REALIZADAS EN 1998. CUERnavaca, Morelos, México 
citologías: se les muestran fotografías de laminillas con tejido sano y no sano para verificar si su interpretación es correcta.

En CIDHAL, el control de calidad es más riguroso. De acuerdo a la información del personal de la organización y del laboratorio al que contratan, en una primera etapa, los patólogos dan una calificación a cada laminilla y reportan las tomas que se deben repetir. En la segunda etapa, CIDHAL elige, periódicamente y de manera aleatoria, una muestra de $10 \%$ de las laminillas ya interpretadas y se envían nuevamente al laboratorio para que las interprete un patólogo distinto al que lo hizo la primera vez. En una tercera etapa se selecciona nuevamente $10 \%$ del total de las laminillas ya interpretadas y se envían al laboratorio central de la ciudad de México. Este último resultado se utiliza como gold standard.

\section{Perspectiva del entrevistador}

El personal de la clínica de la SSA que participa en el PNDC, considera a éste como una de las prioridades de la salud pública en México. Conocen las limitaciones institucionales y las barreras culturales que enfrentan en el intento de alcanzar las metas que establece la SSA en relación con el programa. En este sentido, el personal es muy similar al que colabora en CIDHAL, y esto se refleja en el buen trato que dan ambos proveedores a las usuarias. Sin embargo, en CIDHAL no sólo se tiene conocimiento de esas restricciones, sino que se realizan acciones para superarlas.

Mientras que el personal de la clínica de la SSA espera a que lleguen mujeres a hacerse la prueba, o convencen a quienes llegan por otra causa a consulta a ingresar al programa, en CIDHAL se convence para que se hagan la prueba a mujeres que incluso nunca habían visitado el consultorio. Para lograr esto se programan periódicamente reuniones en la casa de alguna de las parteras, o en alguna clínica de la SSA con la que se haya colaborado, tanto en la ciudad de Cuernavaca como en algunas zonas rurales del estado de Morelos. A cada reunión se invita a mujeres que vivan cerca del lugar para proporcionarles información relacionada con la prevención y detección del cáncer cervicouterino y hacerles la prueba de Papanicolaou.

En contraparte, aunque el personal de salud de la SSA asegura que se tienen pláticas grupales de promoción del programa, no se tuvo acceso a ninguna. Lo que sí se observó es que a cada mujer que acude a consulta, por cualquier causa, se le pregunta en qué fecha se realizó por última vez la prueba, y en caso de no habérsela hecho nunca o sí, pero con más de tres años de anterioridad, se les menciona el riesgo de de- sarrollar cáncer y la importancia de realizarse la prueba lo más pronto posible. En relación con el material y el espacio reconocidos por usuarias y personal de salud, se comprobó la información dada en las entrevistas.

\section{Discusión}

Este estudio es innovador ya que compara el proceso de producción de una intervención de salud preventiva entre dos proveedores de distintos sectores del mercado, el sector público y el sector privado no lucrativo. La información utilizada para realizar el análisis se recopiló entre abril y julio de 1999. Los resultados obtenidos con el análisis de costos y de calidad se refieren a las dos unidades que se incluyeron en el estudio, y en particular a las 30 usuarias que integran el total de la muestra.

Los principales hallazgos del presente estudio fueron: 1) Los costos totales promedio de CIDHAL, inferiores a los de la clínica de primer nivel de la SSA, 2) las usuarias de CIDHAL reconocieron una mayor satisfacción por la atención recibida en comparación con las usuarias de la SSA, 3) el tiempo de espera, previo a la prueba y para recibir resultados, influyó de manera importante en la diferencia de costos y en el nivel de satisfacción aceptado por las usuarias.

Por lo que se refiere al estudio de costos, los costos totales promedio del proceso de producción, desde la perspectiva social, fueron $26 \%$ más altos para la SSA (144 pesos) que para CIDHAL (114 pesos), si se considera el tiempo promedio de espera de las usuarias antes de pasar a la toma de la citología o a recoger los resultados. Es importante resaltar que el tiempo de espera de las usuarias en CIDHAL fue siempre inferior al de las de la clínica de la SSA.

Contrario a lo que se esperaría, por el volumen de laminillas que analiza el laboratorio de la SSA, el costo de producción del proceso de tinción e interpretación de las muestras fue superior en ésta (35 pesos), en comparación con el laboratorio privado (22 pesos). Esto se debe, entre otras cosas, a que en el laboratorio de la SSA el precio de compra de los insumos que se utilizan para la tinción fue más alto: utilizaron más personal y pagaron mejores salarios.

Esto confirma los resultados del estudio de Hernández, ${ }^{14}$ en el que el total de los costos de la toma, traslado, tinción e interpretación y entrega de resultados en la SSA es muy similar. Hernández reportó, en 1997, como costo total 11.56 USD y el resultado obtenido en este estudio es de 12 USD.

En cuanto a la calidad, las usuarias de CIDHAL afirmaron tener mayor satisfacción por la atención re- 
cibida, el espacio disponible para realizar la toma de la citología y la información proporcionada sobre el programa de detección, en comparación con las usuarias de la Clínica de la SSA. La mayor inconformidad de las que se realizaron la prueba en la SSA fue el tiempo de espera previo a la toma y el tiempo de espera por los resultados. Ambos tiempos constituyen un factor importante para la permanencia en el programa: en el primer caso, una larga espera previa a la toma desincentiva a las mujeres a regresar, ya que implica una gran pérdida de tiempo, y en el segundo caso, después de un mes es fácil olvidarse de recoger los resultados.

El control de calidad de la toma e interpretación de las citologías se realiza en ambos lugares, sin embargo, éste es más riguroso en CIDHAL. Tanto en CIDHAL como en la SSA se verifica el estado de la toma y se regresa si estuvo mal tomada. En el caso de CIDHAL hay contacto directo entre los patólogos y el personal que toma la muestra, y cuando alguna laminilla presenta problemas, en una semana, a más tardar, se regresa para repetirla; en el caso de la SSA, el citotecnólogo puede tardar más de un mes en verificar si la toma fue o no adecuada y no tiene contacto directo con el personal encargado de la toma en la clínica. En ambos lugares hay también verificación de la interpretación de las citologías. La cantidad total de laminillas que reinterpretan ambos es muy similar. Sin embargo, el laboratorio de la SSA no realiza una segunda reinterpretación, en el ámbito local, como en el caso de CIDHAL.

No fue posible calcular el nivel de productividad del personal de salud para determinar cuánto tiempo, de las ocho horas que les corresponde trabajar diariamente, realmente lo dedican a la atención de pacientes. De esta forma se tendría un indicador más preciso del tiempo real de trabajo de cada miembro del personal. En un estudio posterior se podría realizar un estudio sombra para estimar el nivel de productividad del personal, y evitar así una subestimación del costo de recursos humanos.

Un elemento importante del desempeño de CIDHAL es la campaña constante que realizan con el fin de convencer a las mujeres para que se hagan la prueba por primera vez. De acuerdo con la información de los cuestionarios aplicados a las usuarias, $20 \%$ de las mujeres de la SSA se practicaron la prueba por primera vez, en CIDHAL fueron $40 \%$. De acuerdo con información proporcionada por la médica responsable del programa en CIDHAL, 25\% de las pruebas realizadas en 1998 fueron de primera vez, de un total de 356 citologías. En contraparte, en la SSA ese mismo año se realizaron sólo 152 pruebas, sin embargo, no se conoce el número de mujeres que se hicieron la prueba por primera vez. Se sugiere, cuando esté disponible, comparar la información de pruebas de primera vez de 1998 con la de 1999, tanto para la SSA como para CIDHAL.

Para profundizar y verificar los hallazgos de este trabajo, se recomienda hacer un estudio de costo-efectividad comparando, también, el trabajo realizado en ambas unidades: la SSA y CIDHAL. Otra recomendación sería estimar un tamaño de muestra que permita presentar resultados representativos de la población que se atiende en ambas unidades y que sean estadísticamente significativos. Conviene mencionar que los resultados obtenidos para la clínica de la SSA, y para CIDHAL, no son representativos de otras clínicas u organizaciones similares.

Podemos concluir que las diferencias en los costos y los indicadores de calidad de la atención entre ambos proveedores no son muy amplias. No obstante, CIDHAL realizó un mejor desempeño al proporcionar atención de mejor calidad sin incrementar los costos. El tiempo de espera, tanto en el análisis de costos como en el de calidad, es el factor que afecta en mayor medida el desempeño de esta clínica, en particular, de la SSA. CIDHAL dio solución al tiempo de espera y proporcionó un mayor número de pruebas. Como ya se mencionó, parte de su trabajo lo realiza en colaboración con algunas clínicas de la SSA pero no la de este estudio. Se sugiere realizar un estudio similar considerando un tamaño de muestra representativo y un estudio de factibilidad política para explorar la posibilidad de colaboración entre distintas ONGs y la SSA dentro del Programa de Detección Oportuna del Cáncer Cervicouterino. Es importante señalar que en ningún momento se propone que CIDHAL pueda suplir el trabajo que realiza la SSA dentro del PNDC, puesto que no cuenta con la infraestructura suficiente.

\section{Agradecimientos}

Agradezco a los doctores Stefano Bertozzi y Gustavo Nigenda por su asesoría; al doctor Octavio Gómez por su paciente revisión de este artículo; a la doctora Guadalupe Mainero, a las parteras y al personal del laboratorio que colaboran con CIDHAL; a la doctora Patricia Mora; a la directora y al personal de salud de la clínica de la SSA de Chipitlán; a la doctora Patricia Alonso y al personal del laboratorio central del Hospital General de México; a la sicóloga Rosario Valdés, al doctor Armando Arredondo; a la doctora Patricia Hernández y al doctor Aurelio Cruz. 


\section{Referencias}

1. Megevand $E, W$ ybrand,$K$ Knight $B, C$ loch B. C an cervical cancer be prevented by see, screen, and treat program?A pilot study.Am J 0 bstet $\mathrm{G}$ ynecol 1996;174(3):923-928.

2.W ilson S,W oodman C,A ssesing effectiveness of cervical screening. C lin O bstet Gynecol 1995;38(3):577-584.

3. Barnum H, Greenberg. Cancer. En: Jamison D, Mosley H, Measham A, Bobadilla JL, comp. Disease control prio rities in developing countries. N ueva York: 0 xford University Press, 1994:540.

4. Brown A, Garber A. Cost-effectiveness of methds to enhance the sensitivity of Papanicolaou testing JAMA 1999;281(4):347-353.

5. Koopmanschp M, Van Ineveld M, Miltenburg T. C osts of home care for advanced breast and cervical cancer in relation to cost-effectiveness of screening. Soc Sci Med 1992;35(8):979-985.

6. Mandelblatt J, Fash M.The cost-effectiveness of cervical cancer screening for low income eldeerly women. JAMA 1988;259(16):2409-2413.

7. Secretaría de Salud. Registro histopatológico de neoplasias en México. México, D.F.: DGE, SSA, 1996.

8. Lazcano-Ponce E, Buiatti E, Nájera P,Alonso P, Hernández M. Evaluation model of the Mexican national program for early cervical cancer detection. Cancer Causes Control 1998;9:241-251.

9. Alonso P, Lazcano E. Q uality control in cytopathology laboratories in six Latin American countries. En:W ied G, Keebler C, Rosenthal D, comp. Compendium on $\mathrm{Q}$ uality Assurance, proficiency testing and work load limitations in clinical citology. Illinois: tutorials of citology Chicago, 1995: 115-121.
10. Meneses $F$, Lazcano $E$, Lino $M$, Hernández $M$, Lezana $M A, N$ ájera $P$, Sepúlveda J. Prevalencia de uso de la prueba de Papanicolaou en mujeres de 15 a 49 años en México. Rev Inst $N$ al. Cancerol (Mex) 1999;45(1): 17-23.

11. Hernández $M$, Lazcano-Ponce $E$, Alonso $P$, Romieu I. Evaluation of the cervical cancer screening programme in Mexico:A population based casecontrol study. Int J Epidemiol 1998;27(3):370-376.

12. Diario 0 ficial. Modificación a la N orma 0 ficial Mexicana N O M-014SSA2-1994 para la prevención, control y tratamiento del cáncer de cuello del útero. México, D.F., 6 marzo de 1998.

13. Lazcano E, N ájera P,Alonso P, Buiatti E, Hernández Avila M. Programa de detección oportuna del cáncer cervical en México I. Diagnóstico Situacional. Rev Inst N ac Cancerol 1996;24(3):123-140.

14. Hernández $P$, Lazcano $E$, Alonso $P, C$ ruz $A$, Meneses $F$, Hernández $M$. Análisis Costo beneficio del programa de detección oportuna de cáncer cervicouterino. Salud Publica Mex 1997;39(4):379-387.

15. Arredondo A, Damián T, De Icaza E. Una aproximación al estudio de costos de servicios de salud en México. Salud Publica Mex 1995;37(5): 437-445.

16. Haddix A, Teustch $S$, Shaffer $P$, D unet D. Prevention effectiveness. A guide to decision analysis and economic evaluation. $N$ ueva York: 0 xford University Press, 1996:67.

17. Drummond M. Methods for the economic evaluation of health care programmes. 2a. edición. N ueva York: 0 xford U niversity Press, 1999:71. 18. Bruce J. Fundamental Elements of the quality of care:A simple framework. Stud Fam Plann 1990; 21: 61-91.

19. Stein K. U sing situation analysis to assess women's perceptions of quality of maternal-child health and family planning services. Reprod Health Matters 1998; 6 (11): 45-54. 